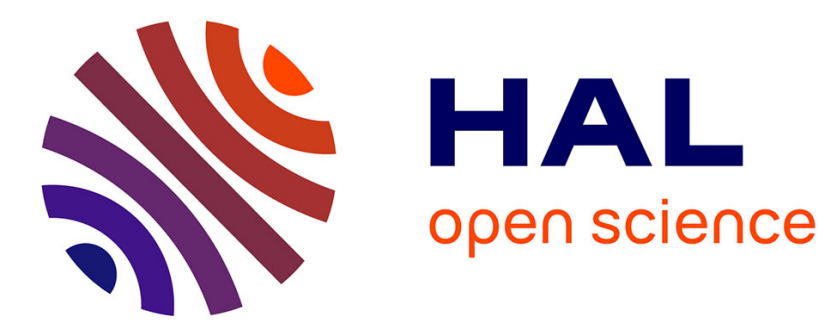

\title{
Externalities in Economies with Endogenous Sharing Rules
}

\author{
Philippe Bich, Rida Laraki
}

\section{To cite this version:}

Philippe Bich, Rida Laraki. Externalities in Economies with Endogenous Sharing Rules. Economic Theory Bulletin, 2017, 5 (2), pp.127-137. 10.1007/s40505-017-0118-3 . halshs-01437507

\section{HAL Id: halshs-01437507 https://shs.hal.science/halshs-01437507}

Submitted on 17 Jan 2017

HAL is a multi-disciplinary open access archive for the deposit and dissemination of scientific research documents, whether they are published or not. The documents may come from teaching and research institutions in France or abroad, or from public or private research centers.
L'archive ouverte pluridisciplinaire HAL, est destinée au dépôt et à la diffusion de documents scientifiques de niveau recherche, publiés ou non, émanant des établissements d'enseignement et de recherche français ou étrangers, des laboratoires publics ou privés. 


\title{
EXTERNALITIES IN ECONOMIES WITH ENDOGENOUS SHARING Rules
}

\author{
PhilipPe Bich, RidA LARAKI ${ }^{\dagger}$
}

January 17, 2017

\begin{abstract}
Endogenous sharing rules were introduced by Simon and Zame [16] to model payoff indeterminacy in discontinuous games. They prove the existence in every compact strategic game of a mixed Nash equilibrium and an associated sharing rule. We extend their result to economies with externalities [1] where, by definition, players are restricted to pure strategies. We also provide a new interpretation of payoff indeterminacy in Simon and Zame's model in terms of preference incompleteness.
\end{abstract}

KeYwords: Abstract Economies, Generalized Games, Endogenous Sharing Rules, Walrasian Equilibrium, Incomplete and Discontinuous Preferences, Better Reply Security

JEL Classification: C02, C62, C72, D50.

\section{Introduction}

The model of strategic games with endogenous sharing rules was introduced by Simon and Zame [16]. Formally, it is a $(N+1)$-tuple $\mathcal{G}=\left(\left(X_{i}\right)_{i \in N}, \mathcal{U}\right)$, where $N$ is the $\operatorname{set}^{1}$ of players, $X_{i}$ is the strategy set of player $i \in N$, and $\mathcal{U}$ is a multivalued function from $X:=\prod_{i \in N} X_{i}$ to $\mathbf{R}^{N}$ with nonempty values. The set $\mathcal{U}(x) \subset \mathbf{R}^{N}$ can be interpreted as the universe of payoff possibilities, given the strategy profile $x \in X$. When $\mathcal{U}(x)=\left\{\left(u_{i}(x)\right)_{i \in N}\right\}$ is a singleton for every $x \in X, \mathcal{G}$ reduces to a usual strategic game, $u_{i}$ being the payoff function of player $i$.

Simon and Zame [16] provide conditions that guarantee the existence of a solution for $\mathcal{G}$, i.e., existence of a selection $q=\left(q_{i}\right)_{i \in N}$ of $\mathcal{U}$ (a sharing rule of $\mathcal{U}$ ), together with a mixed Nash equilibrium $m^{*}=\left(m_{i}^{*}\right)_{i \in N}$ of the game $G=\left(\left(X_{i}\right)_{i \in N},\left(q_{i}\right)_{i \in N}\right)$.

${ }^{*}$ Paris School of Economics, Centre d'Economie de la Sorbonne UMR 8174, Université Paris I Panthéon/ Sorbonne.

${ }^{\dagger}$ Director of Research at CNRS, Université Paris-Dauphine, PSL Research University, Lamsade, 75016 Paris, France. Also affiliated with Department of Economics, Ecole Polytechnique, France. Laraki's work was supported by grants administered by the French National Research Agency as part of the Investissements d'Avenir program (Idex [Grant Agreement No. ANR-11-IDEX-0003-02/Labex ECODEC No. ANR11- LABEX-0047] and ANR-14-CE24-0007-01 CoCoRICo-CoDec).

${ }^{1}$ For simplicity, we use the same letter $\mathrm{N}$ for the set of players or the number of players. 
The concept of sharing rule gives rise to many interpretations. Imagine a designer who must determine who wins an indivisible object in some auction including tie-breaking rules. In that case, selections of $\mathcal{U}$ represent admissible auction rules, and a solution can be seen as a mechanism and a Nash equilibrium of the induced game. Another motivation comes from the payoff indeterminacy that many economic models exhibit: for example, several producers have to choose, each, a location in an area where a continuum of consumers are uniformly distributed. Assume each consumer goes to the closest location. Then payoffs are not well defined when some producers choose the same location: indeed, any division of consumers between the producers is plausible. Simon and Zame's result guarantees the existence of a market sharing rule under which the discontinuous game played by the producers admits a mixed Nash equilibrium.

In this note, we prove existence of a Simon and Zame "solution" in economies with externalities (also called generalized games). This is a general equilibrium model, introduced by Arrow and Debreu [1], in which players play in pure strategies and each player admissible set of strategies is constrained by the strategies chosen by the opponents. For example, in exchange economies, consumers are limited by their budget constraint, which depends on the price vector, itself depending on consumers' demands.

Our second contribution is an interpretation of sharing rules indeterminacy in terms of preference incompleteness. As Aumann [2] argues: "of all axioms of utility theory, the completeness axiom is perhaps the most questionable". Following this seminal paper, many extensions of equilibrium models to incomplete preferences have been investigated, either for continuous preferences $[9,14]$, or discontinuous ones $[6,12,17]$. In this note, we will assume that the ambiguity generated by the indeterminacy of payoffs creates incompleteness in the preferences. This permits to associate to every economy with externalities and endogenous sharing rules an economy with externalities and incomplete and discontinuous preferences. We prove that, in general, this economy does not possess a Nash equilibrium, but it is possible to complete the preferences in a weak sense to restore the existence of an equilibrium.

\section{Economies with Endogenous Sharing Rules}

An Economy with externalities and endogenous sharing rules $\mathcal{E}$ is a pair $\mathcal{E}=(\mathcal{G}, \mathcal{B}=$ $\left.\left(\mathcal{B}_{i}\right)_{i \in N}\right)$ where $\mathcal{G}=\left(\left(X_{i}\right)_{i \in N}, \mathcal{U}\right)$ is a game with endogenous sharing rules, and $\mathcal{B}_{i}$ is a multivalued mapping from $X_{-i}$ to $X_{i}$ with a closed graph and nonempty convex values (i.e., a Kakutani-type mapping).

Definition 1 A solution of $\mathcal{E}$ is a pair $\left(x^{*}, q\right)$, where $q=\left(q_{i}\right)_{i \in N}$ is a selection of $\mathcal{U}$, and $x^{*} \in X$ is a generalized Nash equilibrium of $\left(\left(X_{i}\right)_{i \in N},\left(q_{i}\right)_{i \in N}, \mathcal{B}\right)$, i.e.:

(i) For every $i \in N, x_{i}^{*} \in \mathcal{B}_{i}\left(x_{-i}^{*}\right)$.

(ii) For every $x_{i} \in \mathcal{B}_{i}\left(x_{-i}^{*}\right), q_{i}\left(x_{i}, x_{-i}^{*}\right) \leq q_{i}\left(x^{*}\right)$.

Consider the following assumptions:

$A 1: \quad X$ is a convex and compact subset of a Hausdorff and locally convex topological 
vector space;

$A 2: \mathcal{U}$ is bounded;

$A 3$ : The graph of $\mathcal{E}$, defined by $\Gamma:=\left\{(x, v): v \in \mathcal{U}(x)\right.$ and $x_{i} \in \mathcal{B}_{i}\left(x_{-i}\right)$ for every $\left.i \in N\right\}$, is closed;

A4: $\mathcal{U}$ admits a selection $u=\left(u_{i}\right)_{i \in N}$, such that each $u_{i}$ is quasiconcave in player i's strategy.

Theorem 2 Any economy with externalities and endogenous sharing rules satisfying A1 to A4 admits a solution.

This can be related to Simon and Zame existence result [16]: they prove the existence of a solution in mixed strategies in every strategic games under $A 1, A 2, A 3$ and convexity of $\mathcal{U}(x)$ for every $x \in X$. Theorem 2 proves the existence of a solution in pure strategies, even when the strategies of each player are constrained by the strategies of his opponent. In strategic games where $\mathcal{B}_{i}\left(x_{-i}\right)=X_{i}$ for every $x_{-i} \in X_{-i}$ and every $i \in I$, we get the existence of a solution à la Simon-Zame in pure strategies. This was an open question in Jackson et al. [10] and was solved recently in Bich and Laraki [5] by using as a tool Reny 's [13] better-reply security condition. But we shall see that adding externalities makes the proof more complex. Indeed, the result relies on a recent condition for Nash equilibrium existence in discontinuous games by Barelli and Meneghel [3].

\section{Applications}

\subsection{Incomplete Preferences}

Let us give an interpretation of Theorem 2 in terms of incomplete preferences. If $\mathcal{G}=$ $\left(\left(X_{i}\right)_{i \in N}, \mathcal{U}\right)$ is a game with endogenous sharing rules, then we can define the following preorders $^{2}$ on $X$.

Definition 3 We say that $y \in X$ is $\mathcal{U}$-preferable to $x \in X$ for player $i$, denoted $x \lesssim_{i} y$, if and only if $u_{i}(x) \leq u_{i}(y)$ for every selection ${ }^{3} u$ of $\mathcal{U}$.

When $x$ and $y$ are distinct, $x \lesssim_{i} y$ is equivalent to $\sup \mathcal{U}_{i}(x) \leq \inf \mathcal{U}_{i}(y)$, where $\mathcal{U}_{i}(x)$ denotes the projection of $\mathcal{U}(x) \subset \mathbf{R}^{N}$ on the $i$-th component. In short, $x \lesssim_{i} y$ if and only if $y$ is at least as good as $x$, whatever the indeterminacy of payoffs modeled by $\mathcal{U}$. Formally, to every economy with externalities $\left(\mathcal{G},\left(\mathcal{B}_{i}\right)_{i \in N}\right)$, one can associate an economy with externalities and incomplete preferences $\mathcal{E}=\left(\left(X_{i}\right)_{i \in N},\left(\lesssim_{i}\right)_{i \in N},\left(\mathcal{B}_{i}\right)_{i \in N}\right)$, where the preorders $\lesssim_{i}$ are derived from $\mathcal{U}$ as described above.

\footnotetext{
${ }^{2} \mathrm{~A}$ preorder is a reflexive and transitive binary relation.

${ }^{3}$ Every preorder $\lesssim$ on $X$ admits a multi-utility representation (see [11]), that is there exists a family $\left(v_{j}\right)_{j \in J}$ of real-valued functions defined on $X$ such that: $x \lesssim y \Leftrightarrow$ for every $j \in J, v_{j}(x) \leq v_{j}(y)$. Thus, there is no loss of generality in working with a cardinal multi-representation.
} 
It is then standard to define a generalized Nash equilibrium of $\mathcal{E}$ as a profile $x \in$ $\Pi_{i \in N} \mathcal{B}_{i}\left(x_{-i}\right)$ such that there is no player $i \in N$ and no deviation $y_{i} \in \mathcal{B}_{i}\left(x_{-i}\right)$ with $^{4}$ $x \aleph_{i}\left(y_{i}, x_{-i}\right)$. The following example proves that, in general, $\mathcal{E}$ fails to have a generalized Nash equilibrium, even if the initial game $\mathcal{G}$ satisfies assumptions $A 1$ to $A 4$.

Example 4 Consider a strategic game with endogenous sharing rules and two players. The strategy spaces are $X_{1}=X_{2}=[0,1]$. The endogenous sharing rules are defined by $\mathcal{U}\left(x_{1}, x_{2}\right)=\left(1-x_{1}\left(1-x_{2}\right), 1-\left(1-x_{1}-x_{2}\right)^{2}\right)$ if $\left(x_{1}, x_{2}\right) \neq(0,1)$ and $\mathcal{U}(0,1)=$ $\{(-1,1),(1,1)\}$. This satisfies assumption $A 1$ to $A 4$. In particular, any selection $u$ of $\mathcal{U}$ satisfies the quasiconcavity requirement A4. As described above, this defines a game with incomplete preferences $\mathcal{E}=\left(\left(X_{i}\right)_{i=1,2},\left(\lesssim_{i}\right)_{i=1,2}\right)$. Clearly, for player 2 , the unique bestresponse to $x_{1}$ is $x_{2}=1-x_{1}$. Thus, for every $x_{1}>0,\left(x_{1}, x_{2}\right)$ is not a Nash equilibrium of $\mathcal{E}$, since it would imply $x_{2}=1-x_{1}<1$, but then the only best-response of player 1 is $x_{1}=0$, a contradiction. Thus, the only candidate to be a Nash equilibrium is $(0,1)$, but it is not, since $(0,1) \aleph_{1}(\varepsilon, 1)$ for every $\left.\left.\varepsilon \in\right] 0,1\right]$. Indeed, $1=\sup \mathcal{U}_{1}(0,1) \leq \inf \mathcal{U}_{1}(\varepsilon, 1)=1$ and $1=\sup \mathcal{U}_{1}(\varepsilon, 1)>\inf \mathcal{U}_{1}(0,1)=-1$. Hence, $\mathcal{E}$ has no Nash equilibria. In particular, it is not generalized correspondence secure (see [8]), a condition that would imply the existence of a Nash equilibrium of $\mathcal{E}$.

Thus, one cannot apply recent generalized Nash existence results to $\mathcal{E}$ (e.g., Yannelis, He [17] or Carmona and Podzeck ${ }^{5}$ [8]) simply because the game may fail to have a Nash equilibrium. We now study the possibility of restoring existence after some completion of the preferences. Recall that a completion of the preorder $\lesssim_{i}$ defined on $X$ is a complete preorder $\lesssim_{i}^{\prime}$ on $X$ such that:

(i) $\forall(x, y) \in X^{2}, x \lesssim_{i} y \Rightarrow x \lesssim_{i}^{\prime} y$;

(ii) $\forall(x, y) \in X^{2}, x \aleph_{i} y \Rightarrow x \aleph_{i}^{\prime} y$.

When the preorders $\lesssim_{i}, i \in N$, are defined from $\mathcal{U}$ as above, then for every selection $q$ of $\mathcal{U}$, one can define a $q$-completion of $\lesssim_{i}$ as the complete preorder $\lesssim_{i}^{q}$ on $X$ such that: $x \lesssim_{i}^{q} y \Leftrightarrow q_{i}(x) \leq q_{i}(y)$. This is a weak completion of $\lesssim_{i}$, in the sense that it satisfies property (i) but not property (ii). This is because $x \aleph_{i} y$ is defined by: $v_{i}(x) \leq v_{i}(y)$ for every selection $v$ of $\mathcal{U}$, and $w_{i}(x)<w_{i}(y)$ for at least one selection of $\mathcal{U}$, and this may not imply $q_{i}(x)<q_{i}(y)$.

Corollary 5 Consider an economy with externalities and endogenous sharing rule which satisfies assumptions $A 1$ to $A 4$, and let $\lesssim_{i}$ be the preorders associated to $\mathcal{U}$ as described above. Then there exists a q-completions $\lesssim_{i}^{q}$ of the preorders $\lesssim_{i}(i \in N)$ for some selection $q$ of $\mathcal{U}$, such that $\left(\left(X_{i}\right)_{i \in N},\left(\lesssim_{i}^{q}\right)_{i \in N},\left(\mathcal{B}_{i}\right)_{i \in N}\right)$ has a generalized Nash equilibrium $x^{*} \in X$.

\footnotetext{
${ }^{4}$ Here, $\lesssim_{i}$ denotes the strict preorder associated to $\lesssim_{i}$, that is: for every $(x, y) \in X^{2}, x \lesssim_{i} y$ if and only if $x \lesssim_{i} y$ and not $\left(y \lesssim_{i} x\right)$.

${ }^{5}$ Remark that conversely, our paper does not generalize the existence results of these two papers.
} 
Remark 6 As said above, the completion above may not preserve the strict order. More precisely, it is possible (though not automatic) that the generalized Nash equilibrium $x^{*} \in$ $X$ and the selection $q$ in Corollary 5 satisfy $u_{i}(x) \leq u_{i}(y)$ for every selection $u$ of $\mathcal{U}$ with at least one strict inequality, although $q_{i}(x)=q_{i}(y)$. It is not surprising, since the endogenous sharing rule $q$ can be seen as "summarizing actions taken by unseen agents whose behavior is not modelled explicitely" (see [16]). This unmodeled behaviour implies that the new sharing rule $q$ can really change the preferences of the players (at least at indeterminacy points), and there is no reason why the associated q-completion would preserve the strict order.

Anyway, a possibility to preserve the strict order is to strengthen the definition of the preorders $\widehat{\aleph}_{i}$ as follows. Say that $y \in X$ is $\mathcal{U}$-strongly preferable to $x \in X$ for player $i$, denoted $x \ll_{i} y$, if and only if $u_{i}(x)<u_{i}(y)$ for every selection $u$ of $\mathcal{U}$. This leads to a new notion of profitable deviation for player $i$ (more restrictive than this defined by $\aleph_{i}$ ), thus to a weaker notion of Nash equilibrium. Under assumptions A1 to A4, the existence of a Nash equilibrium for such preorders $\ll_{i}$ is a consequence of Shafer-Sonnenschein's Theorem (see [15]). Indeed, if $y_{i} \in P_{i}(x):=\left\{y_{i} \in X_{i}:\left(x_{i}, x_{-i}\right) \ll_{i}\left(y_{i}, x_{-i}\right)\right\}$, then, by definition of $\ll_{i}$, and because $\mathcal{U}$ has a closed graph, we get $x^{\prime} \ll\left(y_{i}^{\prime}, x_{-i}^{\prime}\right)$ for every $\left(x^{\prime}, y_{i}^{\prime}\right)$ in some neighborhood of $\left(x, y_{i}\right)$. This proves that $P_{i}$ has an open graph. Moreover, $x_{i} \notin c o P_{i}(x)$ for every $i \in I$ (from assumption A4). Consequently, we can apply ShaferSonnenschein's Theorem to get the existence of $x^{*} \in X$ such that $P_{i}\left(x^{*}\right)=\emptyset$ for every $i \in N$, i.e. $x^{*}$ is a Nash equilibrium in the game defined by the preorders $\ll_{i}$. This can be extended to the case of an economy with endogenous sharing rules.

\subsection{Generalized Games with Discontinuous Payoffs}

For every generalized game $\left.G=\left(\left(X_{i}\right)_{i \in N},\left(u_{i}\right)_{i \in N}\right), \mathcal{B}\right)$ where each utility function $u_{i}$ is assumed to be bounded and quasiconcave with respect to $x_{i}$, we can restore existence of a generalized Nash equilibrium by changing the payoff functions at discontinuity points under the constraint that the graph of the new game remains in the closure of the graph of the original game. More precisely, we can construct new payoff functions $q=\left(q_{i}\right)_{i \in N}$ such that:

(a) the economy with externalities $\left.G^{\prime}=\left(\left(X_{i}\right)_{i \in N},\left(q_{i}\right)_{i \in N}\right), \mathcal{B}\right)$ admits a generalized Nash equilibrium $x^{*}$.

(b) for every $y \in X$ with $y_{i} \in \mathcal{B}_{i}\left(y_{-i}\right)$ for every $i \in N$, there is a sequence $\left(y^{n}\right)_{n \in \mathbb{N}}$ converging to $y$ such that $y_{i}^{n} \in \mathcal{B}_{i}\left(y_{-i}^{n}\right)$ for every $i \in N$ and $q(y)=\lim _{n \rightarrow+\infty} u\left(y^{n}\right)$;

This extends the sharing rule existence result in [5] (Theorem 2). The proof is a direct consequence of Theorem 2. Indeed, for every profile $y \in X$, define $\mathcal{U}(y)$ to be the set of limit points of $\left(u\left(y^{n}\right)\right)_{n \in \mathbb{N}}$ for all possible sequences $\left(y^{n}\right)_{n \in \mathbb{N}}$ converging to $y$ and such that $y_{i}^{n} \in \mathcal{B}_{i}\left(y_{-i}^{n}\right)$ for all $i \in N$. Clearly, $\mathcal{U}$ satisfies all the assumptions $A 1$ to $A 4$. Consequently, from Theorem 2 , there is a solution $(x, q)$, which satisfies conditions (a) 
and (b) above.

\subsection{Exchange Economies}

Consider $n$ consumers and $m$ commodities. The initial endowment $e_{i}$ of consumer $i$ is assumed to be an interior point in $\mathbf{R}_{+}^{m}$. Consumer $i$ 's consumption set is equal to $X_{i}=\left\{x_{i} \in \mathbf{R}_{+}^{m}: x_{i} \leq \sum_{j \in N} e_{j}+(1, \ldots, 1)\right\}$.

Following the interpretation of subsection 3.1, consumer's incomplete preferences are assumed to be represented by a multivalued function ${ }^{6} \mathcal{U}_{i}$ from $X_{i}$ to $\mathbf{R}_{+}$with a closed graph, nonempty bounded values on every compact set and admitting at least one quasiconcave selection $u_{i}$. An example, for $m=2$, could be:

$$
\mathcal{U}_{1}\left(x_{1}, x_{2}\right)= \begin{cases}x_{1}+x_{2} & \text { if } x_{1}+x_{2}<2 \\ x_{1}+x_{2}+1 & \text { if } x_{1}+x_{2}>2 \\ {\left[x_{1}+x_{2}, x_{1}+x_{2}+1\right]} & \text { if } x_{1}+x_{2}=2\end{cases}
$$

In this economy, there is a bonus of 1 unit if the consumer has a sufficient quantity of goods, because he may have a substantial benefit if he has more than some minimal level. Moreover, consumer 1's payoff is indetermined when $x_{1}+x_{2}=2$, and there are many ways to complete the preferences .

Under the above assumptions, there exists a selection $\left(q_{i}\right)_{i \in N}$ of $\left(\mathcal{U}_{i}\right)_{i \in N}$ satisfying (a), (b) and (c) below:

(a) the economy $\left\{X_{i}, q_{i}, e_{i}\right\}_{i \in N}$ admits a walrasian equilibrium $\left(x^{*}, p^{*}\right) \in \prod_{i \in N} X_{i} \times$ $\Delta\left(\mathbf{R}_{+}^{m}\right)$, that is: ${ }^{7}$

(1) $\sum_{i \in N} x_{i}^{*} \leq \sum_{i \in N} e_{i}$, and

(2) $x_{i}^{*}$ maximizes the utility function $q_{i}$ of agent $i$ on his budget set $B_{i}\left(p^{*}\right)=\left\{x_{i} \in\right.$ $\left.X_{i}: p^{*} \cdot\left(x_{i}-e_{i}\right) \leq 0\right\}$.

(b) for every $x_{i} \in B_{i}\left(p^{*}\right)$, there is a sequence $\left(x_{i}^{n}, p^{n}\right)_{n \in \mathbb{N}}$ converging to $\left(x_{i}, p^{*}\right)$, with $x_{i}^{n} \in B_{i}\left(p^{n}\right)$, and such that $\lim _{n \rightarrow+\infty} u_{i}\left(x_{i}^{n}\right)=q_{i}\left(x_{i}\right)$.

(c) for every $x_{i} \in X_{i}$ and $x_{i} \notin B_{i}\left(p^{*}\right), q_{i}\left(x_{i}\right)=u_{i}\left(x_{i}\right)$.

Conditions (b) and (c) guarantee that the payoff function $q_{i}$ is not too far from $u_{i}$ (modifications occur only at discontinuity points that are inside the budget set). In particular, $u_{i}\left(x_{i}\right)=q_{i}\left(x_{i}\right)$ if $u_{i}$ is continuous at $x_{i}$. The proof can be found in appendix B. Let us illustrate the result with an example.

\footnotetext{
${ }^{6}$ Here, to simplify the exposition, we do not allow externalities, that is $\mathcal{U}_{i}$ depends only of player i's strategies.

${ }^{7}$ The set $\Delta\left(\mathbf{R}_{+}^{m}\right)$ denotes the unit simplex of $\mathbf{R}_{+}^{m}$.
} 
Example 7 Consider the following walrasian economy with externalities and discontinuous payoffs: $m=2, e_{1}=e_{2}=(1,1)$ and $\mathcal{U}_{1}=\mathcal{U}_{2}$ defined as above in (1). The following payoff functions $u_{1}$ and $u_{2}$ are quasiconcave selections of $\mathcal{U}_{1}=\mathcal{U}_{2}$ :

$$
u_{1}\left(x_{1}, x_{2}\right)=u_{2}\left(x_{1}, x_{2}\right)= \begin{cases}x_{1}+x_{2} & \text { if } x_{1}+x_{2}<2 \\ x_{1}+x_{2}+1 & \text { if } x_{1}+x_{2}>2, \\ \frac{3 x_{1}}{2}+x_{2} & \text { if } x_{1}+x_{2}=2\end{cases}
$$

However, the exchange economy defined by $u_{1}$ and $u_{2}$ has no walrasian equilibrium. Indeed, suppose $p=\left(p_{1}, p_{2}\right)$ is an equilibrium price vector. If $p_{1} \leq p_{2}$, then no consumer demands $x_{1}$, if $p_{1}>p_{2}$ no consumer demands $x_{2}$. Now, if we consider the selection:

$$
q_{1}\left(x_{1}, x_{2}\right)=q_{2}\left(x_{1}, x_{2}\right)= \begin{cases}x_{1}+x_{2} & \text { if } x_{1}+x_{2} \leq 2 \\ x_{1}+x_{2}+1 & \text { if } x_{1}+x_{2}>2\end{cases}
$$

Then $x^{*}=(1,1), p^{*}=(1,1)$ is a walrasian equilibrium.

\section{Appendix A: Proof of Theorem 2}

The proof consists in several steps: first, we turns $G$ into an auxiliary discontinuous strategic game $G^{\prime}$. Second (steps 2 and 3), we prove the existence of a relaxed Nash equilibrium of $G^{\prime}$. This is used to construct in step 4 a sharing rule solution of $G^{\prime}$ that satisfies some desirable properties. Finally, such a sharing rule solution is used to build a solution of $G$. This methodology follows the one developed in [5] and [4] to prove existence of Nash, approximate and sharing rule equilibria in discontinuous games. But it is more complicated because of the externalities. Importantly, the existence results contained in steps 3 and 4 are valid for any quasiconcave compact discontinuous strategic game $\left(\left(X_{i}\right)_{i \in N},\left(u_{i}\right)_{i \in N}\right)$.

By assumption, $\mathcal{U}$ admits a single-valued selection $\phi=\left(\phi_{i}\right)_{i \in I}$ where each $\phi_{i}$ is quasiconcave in player i's strategy.

Step 1. Associate to $G$ a discontinuous game $G^{\prime}$.

Following an idea of Reny [13], we associate to the economy with externalities $G=$ $\left.\left(\left(X_{i}\right)_{i \in N},\left(\phi_{i}\right)_{i \in N}\right), \mathcal{B}\right)$ a strategic game $G^{\prime}$ as follows. Because $\mathcal{U}$ is bounded, there exists $\Lambda \in \mathbf{R}$ such that $\phi_{i}(x) \geq \Lambda+1$ for every $i \in N$ and every profile $x \in X$. The game $G^{\prime}$ has $N$ players. For every $i \in N$, strategy set of player $i$ is $X_{i}$, and his payoff is

$$
u_{i}(x)= \begin{cases}\phi_{i}(x) & \text { if } x_{i} \in \mathcal{B}_{i}\left(x_{-i}\right) \\ \Lambda & \text { otherwise }\end{cases}
$$

These new payoff functions are also quasiconcave.

Step 2. Generalized regularization of payoff functions of $G^{\prime}$.

Throughout this proof, for every $i \in N, x \in X$, and $U$ in $\mathcal{V}\left(x_{-i}\right)$ (the set of open subsets 
of $\left.X_{-i}\right)$, denote by $W_{U}\left(x_{i}, x_{-i}\right)$ the set of Kakutani-type ${ }^{8}$ multivalued mappings $d_{i}$ from $U$ to $X_{i}$ such that $x_{i} \in d_{i}\left(x_{-i}\right)$ for every $x_{-i} \in U$. Let $\underline{\underline{u_{i}}}: X \rightarrow \mathbf{R}$ be the following regularization ${ }^{9}$ of the utility function $u_{i}$

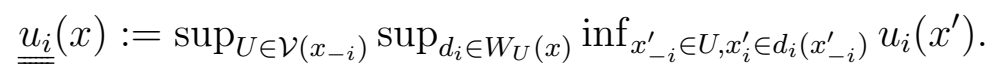

Remark that $\underline{u_{i}}(x) \leq u_{i}(x)$ for every $x \in X$, since in the infimum above one can take $x^{\prime}=x$.

Step 3. Existence of a refined Reny solution of $G^{\prime}$.

Let us prove that there exists a pair $\left(x^{*}, v^{*}\right) \in \bar{\Gamma}$ (where $\Gamma:=\{(x, u(x)): x \in X\}$ ) such that:

$$
\forall i \in N, \sup _{x_{i} \in X_{i}} \underline{\underline{u_{i}}}\left(x_{i}, x_{-i}^{*}\right) \leq v_{i}^{*} .
$$

Such pair $\left(x^{*}, v^{*}\right)$ refines the Reny solution concept introduced in [5]. When $u_{i}$ is continuous for every $i \in N, x^{*}$ is a Nash equilibrium and $v^{*}=u\left(x^{*}\right)$ is the associated payoff vector.

By contradiction, assume that there is no such pair, and let us prove that $G^{\prime}$ is generalized better-reply secure. Recall that $G^{\prime}$ is generalized better-reply secure (Barelli and Meneghel [3]) if whenever $(x, v) \in \bar{\Gamma}$ and $x$ is not a Nash equilibrium, there exists a player $i$ and a triple $\left(d_{i}, V_{x_{-i}}, \alpha_{i}\right)$, where $V_{x_{-i}}$ is an open neighborhood of $x_{-i}, d_{i}$ is a Kakutani-type multivalued function from $V_{x_{-i}}$ to $X_{i}$ and $\alpha_{i}>v_{i}$ is a real number such that for every $x_{-i}^{\prime}$ in $V_{x_{-i}}$ and $x_{i}^{\prime} \in d_{i}\left(x_{-i}^{\prime}\right)$, one has $u_{i}\left(x_{i}^{\prime}, x_{-i}^{\prime}\right) \geq \alpha_{i}$.

For, consider $(x, v) \in \bar{\Gamma}$ such that $x$ is not a Nash equilibrium. By assumption, $(x, v)$ does not satisfy inequality (3), thus there exists some player $i \in N$ such that $\sup _{y_{i} \in X_{i}} \underline{\underline{u_{i}}}\left(y_{i}, x_{-i}\right)>v_{i}$. From the definition of $\underline{\underline{u_{i}}}$, there is $\varepsilon>0, U \in \mathcal{V}\left(x_{-i}\right), d_{i} \in W_{U}(x)$ such that for every $x_{-i}^{\prime} \in U$ and every $x_{i}^{\prime} \in d_{i}\left(x_{-i}^{\prime}\right), u_{i}\left(x_{i}^{\prime}, x_{-i}^{\prime}\right) \geq v_{i}+\varepsilon$ : this implies generalized better-reply security. Consequently, from Barelli and Meneghel [3], since $G^{\prime}$ is generalized better-reply secure, it admits a Nash equilibrium. But this is a contradiction, since if $x \in X$ is a Nash equilibrium, $(x, u(x))$ satisfies inequality (3) (because $\underline{\underline{u_{i}}}(x) \leq u_{i}(x)$ for every $\left.x \in X\right)$. By contradiction, this proves the existence of $\left(x^{*}, v^{*}\right) \in \bar{\Gamma}$ satisfying inequality (3).

Step 4. Existence of a sharing rule solution of $G^{\prime}$.

We now prove that there exists some new payoff functions $\left(q_{i}\right)_{i \in I}$ and a pure Nash equilibrium $x^{*} \in X$ of $G^{\prime \prime}=\left(\left(X_{i}\right)_{i \in N},\left(q_{i}\right)_{i \in N}\right)$, with the additional properties:

(i) for every $i$ and $d_{i} \in X_{i}, q_{i}\left(d_{i}, x^{*}{ }_{-i}\right) \geq \underline{\underline{u_{i}}}\left(d_{i}, x^{*}{ }_{-i}\right)$.

\footnotetext{
${ }^{8} \mathrm{~A}$ Kakutani-type multivalued mapping is a multivalued mapping with nonempty convex values and a closed graph.

${ }^{9}$ This function was introduced by Carmona (see [7]).
} 
(ii) For every $y \in X$, there exists some sequence $\left(y^{n}\right)$ converging to $y$ such that $u\left(y^{n}\right)$ converges to $q(y)$.

For every $i \in N$, denote by $\underline{\underline{\mathcal{S}_{i}}}(y)$ the space of sequences $\left(y^{n}\right)_{n \in \mathbf{N}}$ of $X$ converging to $y$ such that $\lim _{n \rightarrow+\infty} u_{i}\left(y^{n}\right)=\underline{\underline{u_{i}}}(y)$. Then, define $q: X \rightarrow \mathbf{R}^{N}$ by

$q(y)= \begin{cases}v^{*} & \text { if } y=x^{*}, \\ \text { any limit point of } u\left(x^{n}\right)_{n \in \mathbf{N}} & \text { if } y=\left(d_{i}, x_{-i}^{*}\right) \text { for some } i \in N, d_{i} \neq x_{i}^{*},\left(x^{n}\right)_{n \in \mathbf{N}} \in \underline{\underline{\mathcal{S}_{i}}}\left(d_{i}, x_{-i}^{*}\right), \\ q(y)=u(y) & \text { otherwise. }\end{cases}$

Since $\left(x^{*}, v^{*}\right) \in \bar{\Gamma}$, and by definition of $q$, condition (ii) above is satisfied at $x^{*}$. Clearly, by definition, it is also satisfied at every $y$ different from $x^{*}$ for at least two components, and finally also at every $\left(d_{i}, x_{-i}^{*}\right)$ with $d_{i} \neq x_{i}^{*}$ (for some $i \in N$ ), from the definition of $q\left(d_{i}, x_{-i}^{*}\right)$ in this case. Condition (i) is true at every $y$ different from $x^{*}$ for at least two components (from $\underline{\underline{u_{i}}} \leq u_{i}$ ), is true at every $\left(d_{i}, x_{-i}^{*}\right)$ with $d_{i} \neq x_{i}^{*}$ by definition, and is finally true at $x^{*}$ from inequality (3). This ends the proof of Step 4.

Step 5. Existence of a solution of $G$.

Now, we finish the proof of Theorem 2. Take $d_{i} \in \mathcal{B}_{i}\left(x_{-i}^{*}\right) \neq \emptyset$. For every $x_{-i}^{\prime}$ in some neighborhood of $x_{-i}^{*}$ and every $x_{i}^{\prime} \in \mathcal{B}_{i}\left(x_{-i}^{\prime}\right)$, we have, by definition, $u_{i}\left(x_{i}^{\prime}, x_{-i}^{\prime}\right)=$ $\phi_{i}\left(x_{i}^{\prime}, x_{-i}^{\prime}\right) \geq \Lambda+1$. Since $\mathcal{B}_{i}$ is a Kakutani-type mapping, this implies, by definition, $\underline{\underline{u_{i}}}\left(d_{i}, x_{-i}^{*}\right) \geq \Lambda+1$ (where $\underline{\underline{u_{i}}}$ is the regularization of $u_{i}$, defined in the beginning of this proof). Thus, from condition (i) in step 4 above, we get

$$
\forall d_{i} \in \mathcal{B}_{i}\left(x_{-i}^{*}\right), q_{i}\left(d_{i}, x_{-i}^{*}\right) \geq \underline{\underline{u_{i}}}\left(d_{i}, x_{-i}^{*}\right) \geq \Lambda+1 .
$$

Since $x^{*}$ is a Nash equilibrium of $G^{\prime \prime}$, we have:

$$
\forall i \in N, q_{i}\left(x^{*}\right) \geq \sup _{d_{i} \in X_{i}} q_{i}\left(d_{i}, x_{-i}^{*}\right) \geq \Lambda+1 .
$$

From condition (ii) in step 4 above, there is a sequence $\left(x^{n}\right)$ converging to $x^{*}$ such that $u\left(x^{n}\right)$ converges to $q\left(x^{*}\right)$. Since $q_{i}\left(x^{*}\right) \geq \Lambda+1$ for every $i \in N$, we cannot have $u_{i}\left(x^{n}\right)=\Lambda$ for $n$ large enough. Consequently, from the definition of $u_{i}$, we get $u_{i}\left(x^{n}\right)=\phi_{i}\left(x^{n}\right)$ and $x_{i}^{n} \in \mathcal{B}_{i}\left(x_{-i}^{n}\right)$ for $n$ large enough. Passing to the limit, we get $x_{i}^{*} \in \mathcal{B}_{i}\left(x_{-i}^{*}\right)$ for every $i \in I$ (because $\mathcal{B}_{i}$ has a closed graph). A similar argument can be applied to any $\left(y_{i}, x_{-i}^{*}\right) \in X$ for which $y_{i} \in \mathcal{B}_{i}\left(x_{-i}^{*}\right)$ : there is a sequence $\left(x^{n}\right)$ converging to $\left(y_{i}, x_{-i}^{*}\right)$ such that $u\left(x^{n}\right)$ converges to $q\left(y_{i}, x_{-i}^{*}\right)$. Since $q_{i}\left(y_{i}, x_{-i}^{*}\right) \geq \Lambda+1$ (from inequality (4)), we cannot have $u_{i}\left(x^{n}\right)=\Lambda$ for $n$ large enough. Consequently, $u_{i}\left(x^{n}\right)=\phi_{i}\left(x^{n}\right)$ and $x_{i}^{n} \in \mathcal{B}_{i}\left(x_{-i}^{n}\right)$ for $n$ large enough. In particular, since $\phi$ is a selection of $U$ and since $U$ has a closed graph, we get

$$
\forall y_{i} \in \mathcal{B}_{i}\left(x_{-i}^{*}\right), q\left(y_{i}, x_{-i}^{*}\right) \in U\left(y_{i}, x_{-i}^{*}\right)
$$

Now, define $\tilde{q}\left(y_{i}, x_{-i}^{*}\right)=q\left(y_{i}, x_{-i}^{*}\right)$ whenever $y_{i} \in \mathcal{B}_{i}\left(x_{-i}^{*}\right)$ for some $i \in N$, and $\tilde{q}(y)=\phi(y)$ elsewhere. The proof that $x^{*}$ is a equilibrium of $\left(\left(X_{i}\right)_{i \in N},\left(\tilde{q}_{i}\right)_{i \in N}, B\right)$ is a straightforward 
consequence of $x^{*}$ being a Nash equilibrium of $\left(\left(X_{i}\right)_{i \in N},\left(q_{i}\right)_{i \in N}\right)$. Last, we have to prove that $\tilde{q}(y) \in U(y)$ for every $y \in X$. This is clear at $y=\left(y_{i}, x_{-i}^{*}\right)$ for $y_{i} \in \mathcal{B}_{i}\left(x_{-i}^{*}\right)$, from (5) above. For others $y$, we have $\tilde{q}(y)=\phi(y) \in U(y)$ by definition. This ends the proof of Theorem 2.

\section{$5 \quad$ Appendix B: proof of the statements in Section 3.3}

From the exchange economy, define an economy with externalities and discontinuous payoffs $(\mathcal{G}, \mathcal{B})$ as follows:

1. There are $(N+1)$ players.

2. For $i=1, \ldots, N$, player $i$ 's convex compact strategy space is $X_{i}=\left\{x_{i} \in \mathbf{R}_{+}^{m}: x_{i} \leq\right.$ $\left.\sum_{i=1}^{N} e_{i}+(1, \ldots, 1)\right\}$ and his payoff function is $u_{i}$.

3. The strategy space of player $(N+1)$ (called the auctioneer) is $X_{N+1}=\Delta\left(\mathbf{R}_{+}^{m}\right)$, and his payoff function is $v_{N+1}(x, p)=p \cdot \sum_{i \in N}\left(x_{i}-e_{i}\right)$.

4. Last, define the strategy correspondences as follows: for every $i \in N, \mathcal{B}_{i}(x, p)=$ $B_{i}(p)=\left\{x_{i} \in X_{i}: p \cdot x_{i} \leq p \cdot e_{i}\right\}$, and finally define $\mathcal{B}_{N+1}(x, p)=X_{N+1}$.

Following section 3.2 , this economy has a solution $\left(x^{*}, p^{*}, \tilde{q}\right)$. This means that:

1. For every $(x, p) \in \prod_{i} X_{i} \times \Delta\left(\mathbf{R}_{+}^{m}\right)$ such that $x_{i} \in B_{i}(p)$ for every $i \in N$, there is a sequence $\left(x^{n}, p^{n}\right)_{n \in \mathbb{N}}$ converging to $(x, p)$ such that $x_{i}^{n} \in B_{i}\left(p^{n}\right)$ for every $i \in N$ and $\tilde{q}_{i}(x, p)=\lim _{n \rightarrow+\infty} u_{i}\left(x_{i}^{n}\right)$. In particular, from the continuity of $v_{N+1}$, $\tilde{q}_{N+1}(x, p)=v_{N+1}(x, p)=p \cdot \sum_{i \in N}\left(x_{i}-e_{i}\right)$.

2. (i) For every $i \in N, x_{i}^{*} \in B_{i}\left(p^{*}\right)$.

(ii) For every $i \in N$, for every $x_{i} \in B_{i}\left(p^{*}\right), \tilde{q}_{i}\left(x_{i}, x_{-i}^{*}, p^{*}\right) \leq \tilde{q}_{i}\left(x^{*}, p^{*}\right)$.

(iii) For every $p \in \Delta\left(\mathbf{R}_{+}^{m}\right), p \cdot \sum_{i \in N}\left(x_{i}^{*}-e_{i}\right) \leq p^{*} \cdot \sum_{i \in N}\left(x_{i}^{*}-e_{i}\right)$.

Let us now define $q_{i}\left(x_{i}\right):=\tilde{q}_{i}\left(x_{i}, x_{-i}^{*}, p^{*}\right)$ for every $x_{i} \in B_{i}\left(p^{*}\right)$, and $q_{i}\left(x_{i}\right)=u_{i}\left(x_{i}\right)$ otherwise. From 1 and 2 above, there is a sequence $\left(x^{n}, p^{n}\right)_{n \in \mathbb{N}}$ converging to $\left(x_{i}, x_{-i}^{*}, p^{*}\right)$ such that $x_{i}^{n} \in B_{i}\left(p^{n}\right)$ for every $i \in N$ and

$$
\tilde{q}_{i}\left(x_{i}, x_{-i}^{*}, p^{*}\right)=\lim _{n \rightarrow+\infty} u_{i}\left(x_{i}^{n}\right)=q_{i}\left(x_{i}\right)
$$

Thus condition (b) and (c) in section 3.3 hold. Let us prove that condition (a) also holds, that is, $\left(x^{*}, p^{*}\right)$ is a walrasian equilibrium of the economy with payoff functions $\left(q_{i}\right)_{i \in N}$. 
First, assume that we do not have $\sum_{i \in N}\left(x_{i}^{*}-e_{i}\right) \leq 0$. Then, let us define $p=$ $(p(1), \ldots, p(k), \ldots, p(m)) \in \Delta\left(\mathbf{R}_{+}^{m}\right)$ with $p(k)=0$ when $\sum_{i \in N}\left(x_{i}^{*}-e_{i}\right)(k) \leq 0$ (where $\sum_{i \in N}\left(x_{i}^{*}-e_{i}\right)(k)$ denotes $k$-component of $\left.\sum_{i \in N}\left(x_{i}^{*}-e_{i}\right)\right)$, and $p(k)=\lambda . \sum_{i \in N}\left(x_{i}^{*}-e_{i}\right)(k)$ otherwise (where $\lambda>0$ is a normalization coefficient that insures that $p \in \Delta\left(\mathbf{R}_{+}^{m}\right)$ ). By definition, we get $p \cdot \sum_{i \in N}\left(x_{i}^{*}-e_{i}\right)>0$, thus from (iii) above, $p^{*} \cdot \sum_{i \in N}\left(x_{i}^{*}-e_{i}\right)>0$. But from condition (i) above, the budget constraint yields $p^{*}\left(x_{i}^{*}-e_{i}\right) \leq 0$ for every $i \in N$, and summing these inequalities, we get $p^{*} \cdot \sum_{i \in N}\left(x_{i}^{*}-e_{i}\right) \leq 0$, a contradiction. This proves $\sum_{i \in N}\left(x_{i}^{*}-e_{i}\right) \leq 0$.

From (ii) above, for every $x_{i} \in B_{i}\left(p^{*}\right)$, we have $q_{i}\left(x_{i}\right)=\tilde{q}_{i}\left(x_{i}, x_{-i}^{*}, p^{*}\right) \leq \tilde{q}_{i}\left(x^{*}, p^{*}\right)=$ $q_{i}\left(x_{i}^{*}\right)$. Thus, for every $i \in N, x_{i}^{*}$ maximizes $q_{i}$ in $B_{i}\left(p^{*}\right)$, which ends the proof.

\section{References}

[1] K.J. Arrow and G Debreu. Existence of an Equilibrium for a Competitive Economy. Econometrica, 22(3):265-290, 1954.

[2] Robert J. Aumann. Utility theory without the completeness axiom. Econometrica, 30(3):445-462, 1962.

[3] Paulo Barelli and Idione Meneghel. A Note on the Equilibrium Existence Problem in Discontinuous Games. Econometrica, 81(2):813-824, 2013.

[4] Philippe Bich and Rida Laraki. A unified approach to equilibrium existence in discontinuous strategic games. Preprint: halshs-00717135, 2012.

[5] Philippe Bich and Rida Laraki. On the existence of approximate equilibria and sharing rule solutions in discontinuous games. Theoritical economics, (accepted) 2016.

[6] Guilherme Carmona. An Existence Result for Discontinuous Games. Journal of Economic Theory, 144(3):1333-1340, 2009.

[7] Guilherme Carmona. Understanding some recent existence results for discontinuous games. Economic Theory, 48(1):31-45, 2011.

[8] Guilherme Carmona and Konrad Podczeck. Existence of Nash Equilibrium in Ordinal Games with Discontinuous Preferences. Working paper, University of Surrey and Universität Wien, 2015.

[9] D. Gale and A. Mas-Colell. An equilibrium existence theorem for a general model without ordered preferences. Journal of Mathematical Economics, 2(1):9-15, March 1975 .

[10] Matthew O. Jackson, Leo K. Simon, Jeroen M. Swinkels, and William R. Zame. Communication and Equilibrium in Discontinuous Games of Incomplete Information. Econometrica, 70(5):1711-1740, 2002. 
[11] Özgür Evren and Efe A. Ok. On the multi-utility representation of preference relations. Journal of Mathematical Economics, 47(4-5):554 - 563, 2011.

[12] P. J. Reny. Nash equilibrium in discontinuous games, working paper. working paper, University of Chicago, 2013.

[13] Philip J. Reny. On the Existence of Pure and Mixed Strategy Nash Equilibria in Discontinuous Games. Econometrica, 67(5):1029-1056, 1999.

[14] Wayne Shafer and Hugo Sonnenschein. Equilibrium in abstract economies without ordered preferences. Journal of Mathematical Economics, 2(3):345 - 348, 1975.

[15] Wayne Shafer and Hugo Sonnenschein. Equilibrium in abstract economies without ordered preferences. Journal of Mathematical Economics, 2(3):345 - 348, 1975.

[16] Leo K Simon and William R Zame. Discontinuous Games and Endogenous Sharing Rules. Econometrica, 58(4):861-872, July 1990.

[17] Nicholas C. Yannelis and Wei He. Equilibrium Theory with Discontinuous and NonOrdered Preferences. Working paper, University of Iowa, 2014. 